\title{
Designing of Ultrasonic Horns to Improve Amplitude Uniformity in Ultrasonic Metal Welding
}

\author{
Yeon Ju Lee ${ }^{1}$, Muhammad Bilal Shahid ${ }^{1}$ and Dong Sam Park ${ }^{1}$ \\ ${ }^{1}$ Dept. of Mechanical Engineering, Incheon National Univ., Yeonsu Gu, Incheon, 22012, Korea
}

\begin{abstract}
The ultrasonic metal welding has been used extensively in many fields because it can weld thin layers of highly conductive and dissimilar materials with high reliability. In particular, one-wave length horns with four welding tips on both sides of the horn are widely used for welding positive and negative electrodes and tabs of rechargeable lithium ion batteries. As the vibrational amplitude of each tip is known to be non-uniform, this causes some problems in welding performance. In this study, the one-wave length horn which has four welding tips on both sides of the horn is newly designed to improve amplitude uniformity of each tip. The design of experiment technique is employed to determine the optimum configuration of the horn. The modal analysis and harmonic response analysis are repeatedly conducted to obtain the uniform amplitude of horn tips, and stress distribution in the horn is analyzed finally. It is shown that the uniformity of amplitude can be improved by making appropriate grooves on both sides of the conventional horn.
\end{abstract}

\section{Introduction}

With the recent expansion of mobile devices, such as smart phones, tablets and PCs, and the development of electric cars, many researches are being carried out actively on their key component, the secondary cell battery. These batteries are supposed to be small, lightweight, and high-capacity. Furthermore, the structure of the battery must be multi-layered. Ultrasonic metal welding has been used for efficient welding of multi-layer tabs.

Most of the studies that have been published are for ultrasonic welding between homogeneous or dissimilar metals [1]. For welding of multiple foil-layers onto a single tab, there is not much research available. So, this topic should be studied in detail. The one-wave length horn which has four welding tips has been widely used to weld positive/negative electrodes and tabs of the rechargeable lithium-ion batteries [2]. The vibrational amplitude of each tip is known to be non-uniform, and this causes some problems in welding performance. Therefore, the differences in amplitude between the tips should be minimized for efficient ultrasonic metal welding.

In past studies associated with this topic, there is a study that reduces the degree of uniformity error that occurs with molded areas during vibratory plate ultrasound of speakers. The vibration characteristics were analyzed by changing the design to improve the uniformity of the output[3]. There is another study that analyzes the effects of grooves and slots on the amplitude uniformity and determines the dimensions of the grooves and slots for high uniformity[4].

In this study, the one-wave length horn which has four welding tips is newly designed to improve vibrational uniformity on each tip of the horn. The design of experiment technique is employed to determine the optimum configuration of the horn. The modal and harmonic response analysis are performed to obtain the uniform amplitude of designed horn tips, and the stress distribution in the horn is analyzed finally. Several different modifications in the shape of conventional horn are tried, and then analyzed by using ANSYS tool.

\section{Shape design of horns}

\subsection{Conventional ultrasonic horns}

This study aims to improve weld quality by reducing differences in amplitude between two central tips and two outer tips, which are generated during welding process with conventional ultrasonic welding horns. Fig. 1 shows the ultrasonic welding horn that is being used in industry.

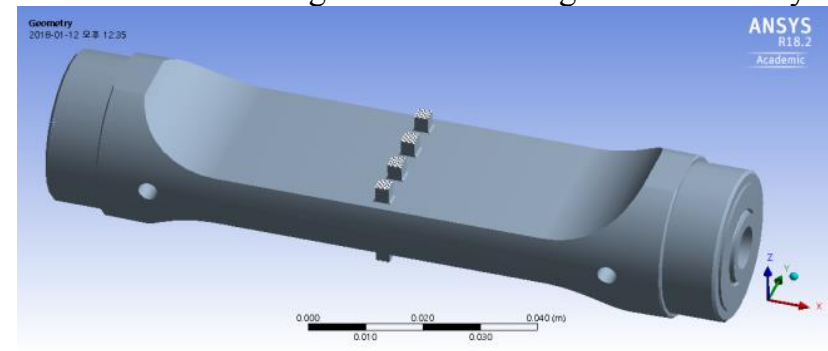

Figure 1. 3D model of a conventional horn

The shape of the horn on which this study is based is a one-wave length horn, designed symmetrically to provide 
stable welding [5]. The required working frequency of the ultrasonic device is $40 \mathrm{kHz}( \pm 500 \mathrm{~Hz})$ and the device will not operate if the frequency fluctuates beyond the suggested tolerance. Therefore, the natural frequency must be within this range while designing horn. The material of the horn is CPM10 V, which has high hardness and excellent vibration characteristics. Table 1 shows the material properties [6].

Table 1. Mechanical properties of horn material, CPM10V

\begin{tabular}{ccc}
\hline \hline Density & Poisson's Ratio & Young's Modulus \\
\hline $0.268\left[\mathrm{lb} / \mathrm{in}^{3}\right]$ & 0.28 & $221[\mathrm{GPa}]$ \\
\hline \hline
\end{tabular}

\subsection{Shape modifying based on uniformity analysis}

In the analysis result of the conventional horn, there are differences in amplitude between two outer tips and two center tips, which cause differences in the weld strength at the point of each tip. It makes adverse effect to weldability so the shape of the horn should be modified to prevent it.

Initially, as shown in Fig. 2, A(14mm), B(2.4mm) C(R15) and $\mathrm{D}(\mathrm{R} 3)$ has been chosen as starting values for analysis. With each of the values of A, B, C, and D being varied, only one at a time, the uniformity is measured and compared with the initial model. To check the uniformity, as shown in Fig. 3, the displacements of three points per tip are measured.

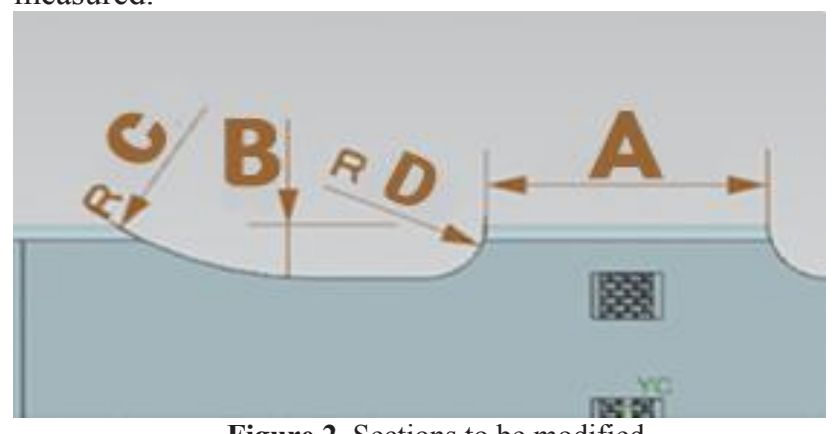

Figure 2. Sections to be modified

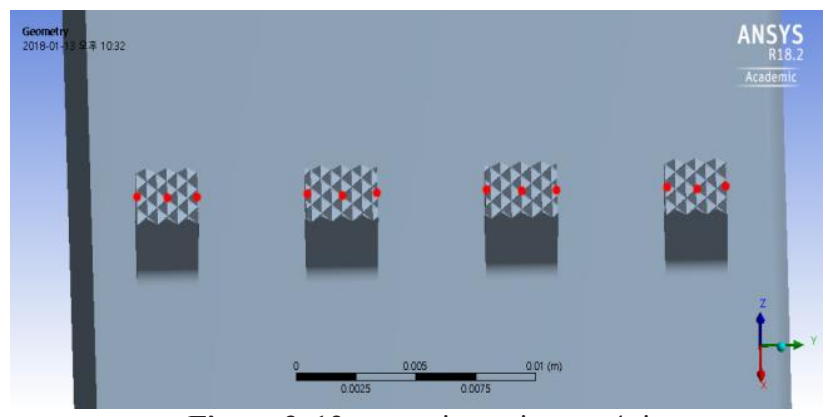

Figure 3. 12 measuring points on 4 tips

The modal analysis was done by increasing the length of part A from $12 \mathrm{~mm}$ to $16 \mathrm{~mm}$. Fig. 4(a) is a graph showing the displacement of each tip. As the most uniform point is found at $14 \mathrm{~mm}(39,638 \mathrm{~Hz})$ shown by the green line on graph. This length is selected as our design point. The same analysis was done by increasing the length of part B from $2.2 \mathrm{~mm}$ to $3 \mathrm{~mm}$. Fig. 4 (b) is a graph showing the displacement of each tip corresponding to different lengths of part B. By changing the radius values from 2.2 to 3.0 $\mathrm{mm}$, the most suitable radius value came out to be $3.0 \mathrm{~mm}$. However, the length of B could not be increased beyond $3 \mathrm{~mm}$, because the stress concentration would increase too much.

The same analysis has been carried out for different radius of C and D. As shown as Fig. 5(a), the most suitable radius for $\mathrm{C}$ was found out to be $\mathrm{R} 18 \mathrm{~mm}(39,683 \mathrm{~Hz})$ with the most uniform displacement (purple line). In the graph on Fig. 5(b), R3 $(39,683 \mathrm{~Hz})$ was selected as the desired radius owing to the best uniformity at that radius (green line).

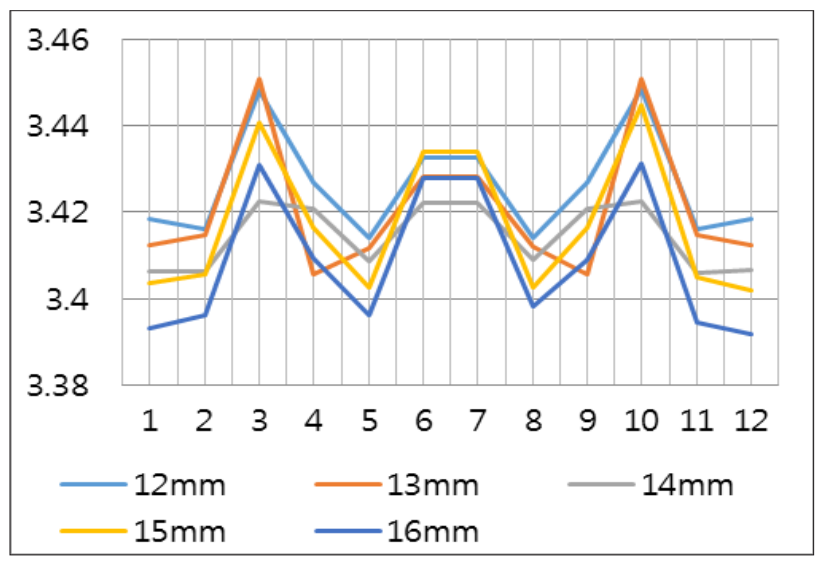

(a) A-length

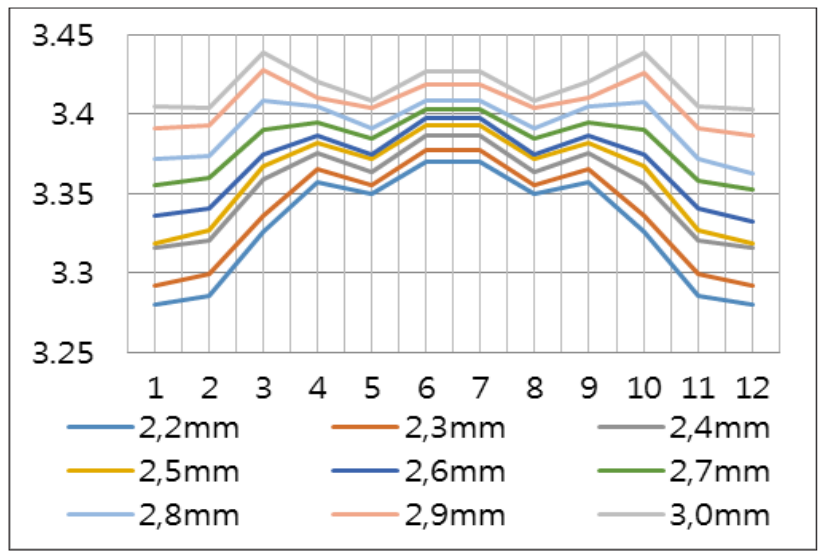

(b) B-length

Figure 4. Effects of the length of $A$ and $B$ on the amplitude variations on the tips

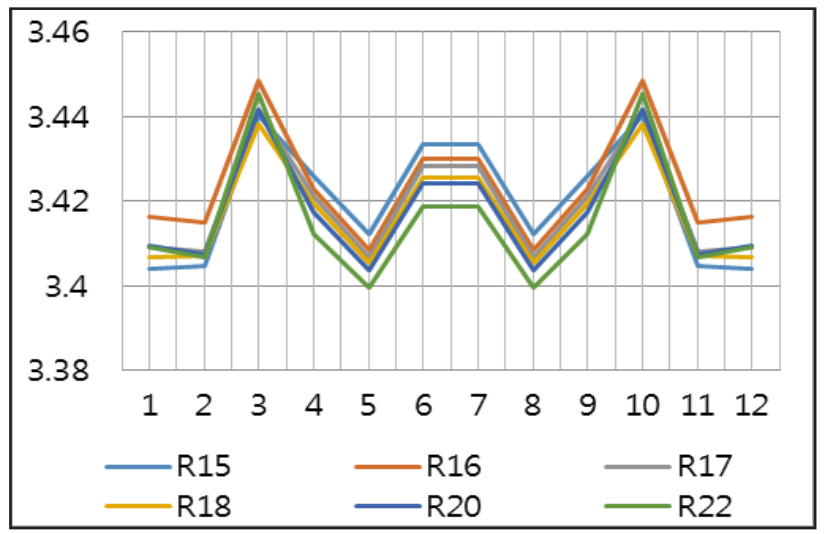

(a) C-radius 


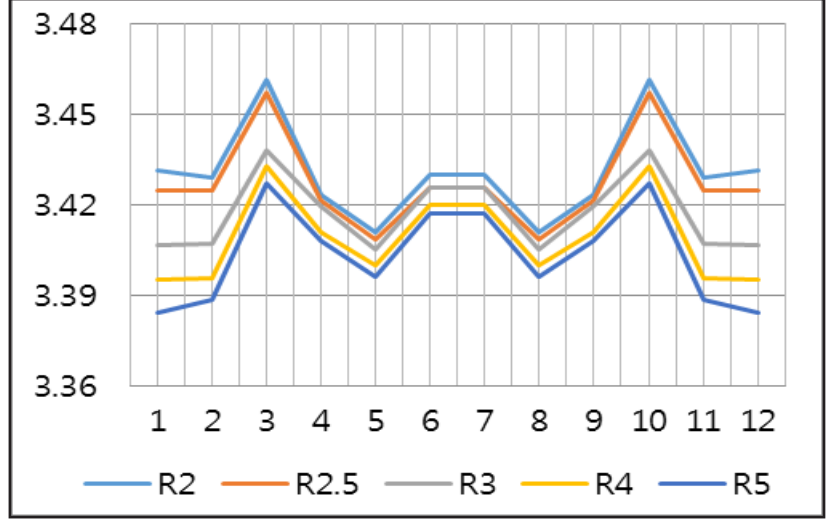

(b) D-radius

Figure 5. Effects of the radius of $\mathrm{C}$ and $\mathrm{D}$ on the amplitude variations on the tips

\section{Vibration and stress analysis}

\subsection{Modal analysis}

To design the new horn, modal analysis is performed using ANSYS tool. Fig. 6 shows non-longitudinal mode $\left(13^{\text {th }}\right.$, $\left.15^{\text {th }}\right)$ adjacent to the longitudinal mode $\left(14^{\text {th }}\right)$. These non-longitudinal vibration modes will adversely affect ultrasonic welding, so it is not suitable for ultrasonic welding that requires the longitudinal vibration mode [7]. The measured frequency in the 14th mode was found to be $39,633 \mathrm{~Hz}$ with an error of approximately $0.92 \%$. The vibration mode is also longitudinal and can be seen to have the maximum amplitude on the center part of horn.

Fig. 7 shows the amplitudes on the tips of horn before (Fig.7 (a)) and after improvement (Fig.7 (b)). As can be seen clearly, higher amplitude (approx. $16.9 \mu \mathrm{m}$ ) occurs on tips of the horn on the right one of Fig. 7 i.e. after improvement. Also, the amplitude distribution in the central tips and the outer tips is more uniform after improvement.

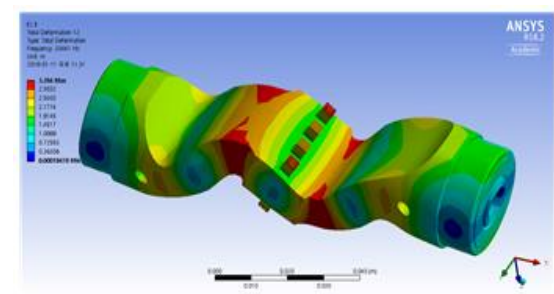

(a) $13^{\text {th }}$ mode $(37,390 \mathrm{~Hz})$

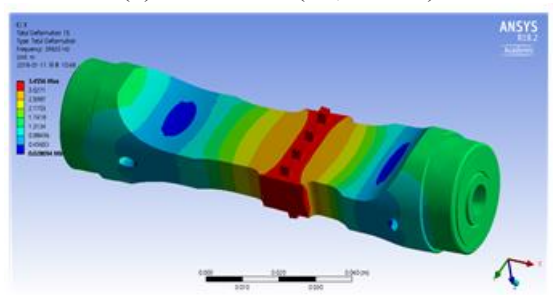

(b) $14^{\text {th }}$ mode $(39,633 \mathrm{~Hz})$

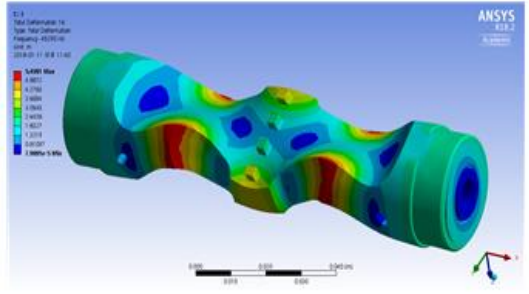

(c) $15^{\text {th }}$ mode $(45,290 \mathrm{~Hz})$

Figure 6. Vibration modes and natural frequencies of the improved horn

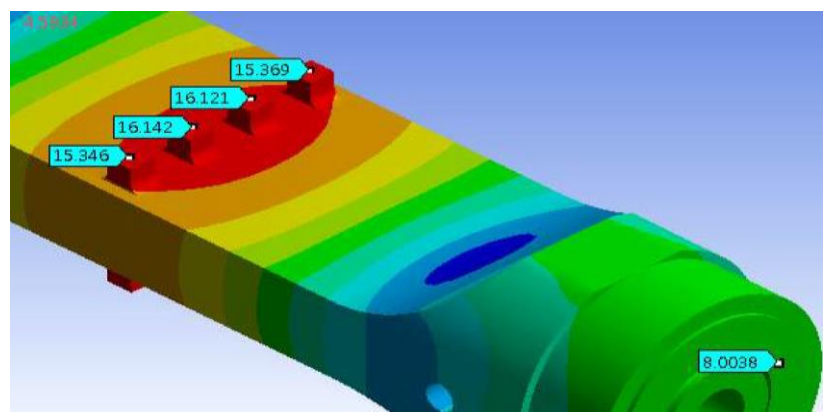

(a) Before improvement

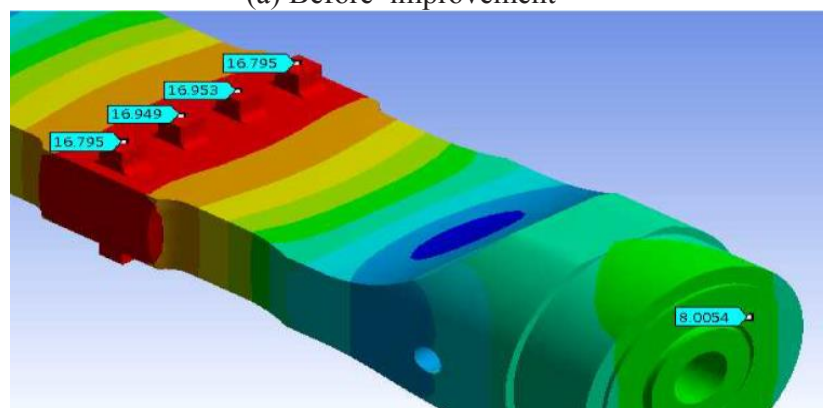

(b) After improvement

Figure 7. Amplitude of horn before/after improvement

\subsection{Harmonic response analysis}

A frequency range of at least $30,000 \mathrm{~Hz}$ to $50,000 \mathrm{~Hz}$ is specified for the input surfaces that meet the booster of the ultrasonic welding tool. As a result of the harmonic response analysis to the knurling part of the tip on the output surface, the peak frequency is found to be $39.6 \mathrm{kHz}$ as shown in Fig.8, which corroborates the results of the modal analysis [8].

\subsection{Stress analysis}

There was no significant difference between the frequencies of the two models before and after the improvements $(39,710 \mathrm{~Hz})$. However, as shown in Fig. 9, the maximum stress in the horn was reduced to some extent, which would improve the life of the horn. It was found that the maximum stress was $215.24 \mathrm{MPa}$ before the improvement, and after the improvement, the stress level was reduced to $185.4 \mathrm{MPa}$. 


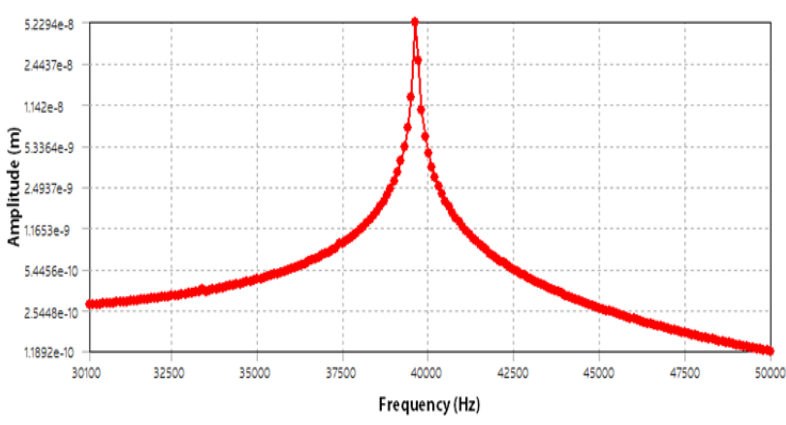

Figure 8. Harmonic response analysis after improvement

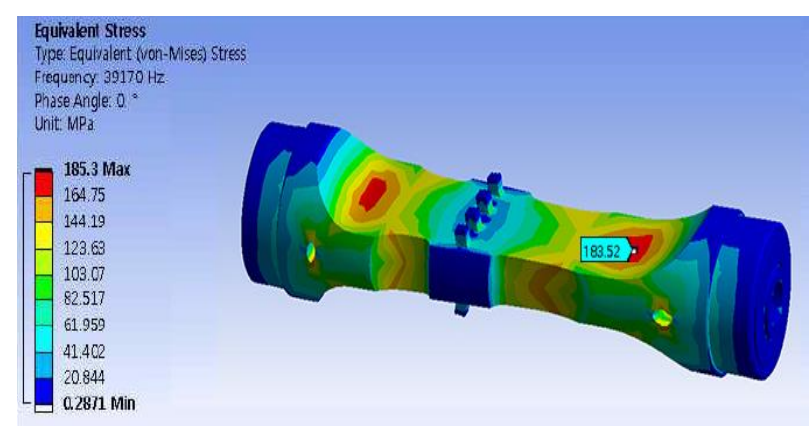

Figure 9. Stress distribution after improvement

\section{Summary}

Recently, ultrasonic horns of one-wave length which have four welding tips have been widely used to weld positive/negative electrodes and tabs of the rechargeable lithium-ion batteries. The vibrational amplitude of each tip is known to be non-uniform, and this causes the some problems in welding performance. In this study, conventional horn of the one-wave length which has four welding tips is modified and designed newly to improve vibrational uniformity of each tip of the horn. The design of experiment technique is employed to determine the optimum configuration of the horn. The modal analysis and harmonic response analysis are conducted to obtain the uniform amplitude of designed horn tips, and stress distribution in the horn is analyzed finally. It is concluded that the uniformity of vibrational amplitude can be improved by making proper grooves on the both sides of the conventional horn. There was no significant difference between the frequencies of the two models before and after the improvements. However, the maximum stress in the horn was reduced to some extents, which could improve the life of the horn. It was found that the maximum stress was $215.24 \mathrm{MPa}$ before the improvement, and after the improvement, the stress level was reduced to $185.4 \mathrm{MPa}$.

\section{Acknowledgement}

This research was supported by Basic Science Research Program through the National Research Foundation of Korea (NRF) funded by the Ministry of Education (Grant No. 2017R1D1A1B03034483).

\section{References}

1. Ho-Su Jang, Woo Yeol Park and Dong-Sam Park, "Design and weld ability verification of the $40 \mathrm{kHz}$ horn for ultrasonic metal welding," Journal of the Korean Society of Manufacturing Process Engineers, vol. 12 (2013), pp. 55-60.

2. Jeong-Seok Seo, Sung-Min Jang and Si-Young Beck, " One-wavelength ultrasonic horn design for ultrasonic machining of mobile phone battery terminal welding," Journal of the Korean Society of Manufacturing Technology Engineers, Vol. 21(2012), pp. 70-75.

3. H.J.Lee, Y.E.Lim and K.Park, "Optimal design of ultrasonic horn for thermoforming process," Journal of Korean Society of Precision Engineering (2016), pp. 234-234.

4. S.R.Kim, C.D.Yoo and J.H.Lee, "Design of ultrasonic horn to enhance amplitude uniformity," Journal of Korean Society of Precision Engineering (2009), pp. 691-692.

5. Jeong-Seok Seo, Yoon-Jung Lee, Si-Young Beck and Dong-Sam Park, "Prediction of crack initiation and design of $40 \mathrm{kHz}$ blade horn for ultrasonic cutting," Journal of the Korean Society of Manufacturing Technology Engineers, Vol. 21 (2012), pp. 784-789.

6. S.R Kim, J.H Lee and Choong D. Yoo, "Design of cylinder horn for ultrasonic welding," Journal of KWJS, vol. 27, No.4 (2009), pp. 60-66.

7. K.Park, W.S.Jung and J.M.Ra, "Optimal design of a tool horn for micro-pattern forming using ultrasonic wave," KSMTE Annual Spring Conference (2012), pp. 47-47.

8. Jeong-Seok Seo, Yoon-Jung Lee, Jin-Wook Kim and Dong-Sam Park, "Design improvement and performance evaluation of $20 \mathrm{kHz}$ horn for ultrasonic welding," Journal of Korean Society of Manufacturing Process Engineers, vol.12, No.5 (2013), pp.135-140. 